\title{
Severe acute toxicity following gemcitabine administration: A report of four cases with cytidine deaminase polymorphisms evaluation
}

\author{
BEATA HRYCIUK $^{1}$, BARTOSZ SZYMANOWSKI ${ }^{1}$, ANNA ROMANOWSKA ${ }^{2}$, EWA SALT $^{1}$, BARTOSZ WASAGG $^{3}$, \\ BARTŁOMIEJ GRALA ${ }^{4}$, JACEK JASSEM ${ }^{2}$ and RENATA DUCHNOWSKA ${ }^{1}$ \\ ${ }^{1}$ Department of Oncology, Military Institute of Medicine, 04-141 Warsaw; Departments of ${ }^{2}$ Oncology and Radiotherapy, \\ and ${ }^{3}$ Biology and Genetics, Medical University of Gdańsk, 80-211 Gdańsk; ${ }^{4}$ Department of Pathology, \\ Military Institute of Medicine, 04-141 Warsaw, Poland
}

Received May 22, 2017; Accepted November 6, 2017

DOI: 10.3892/ol.2017.7473

\begin{abstract}
Gemcitabine (GCB) is a pyrimidine antimetabolite widely used in various solid tumors as a single agent or as a component of multidrug regimens. In the majority of patients, GCB is well tolerated, however life-threatening complications occasionally occur. The current report presents four cases of severe acute toxicity, which included two that were fatal, following administration of GCB alone or in combination with cisplatin. Of the four cases, in one, a Naranjo Adverse Drug Reaction Probability Score was definite, in two, probable and in one possible. To determine the potential causes of these toxicities, polymorphic variants of cytidine deaminase, the primary enzyme involved in the hepatic metabolism of GCB, were assessed. The homogeneous c.435TT variant was detected in one patient and a heterozygotic c.435CT variant in two, one of whom additionally harbored a heterozygotic c.79AC variant.
\end{abstract}

\section{Introduction}

Gemcitabine (GCB) is an anticancer agent widely used alone or in combination with other cytotoxics in the treatment of various malignancies including non-small cell lung cancer (NSCLC), pancreatic, bladder, breast, ovarian, prostate cancer, and cholangiocarcinoma (1). GCB toxicity is generally mild, transitory and rarely dose limiting. Most common side effects include laboratory alterations, such as myelosupression, transaminase elevation, mild proteinuria and hematuria, whereas symptomatic toxicities are usually well controlled and not life

Correspondence to: Dr Renata Duchnowska, Department of Oncology, Military Institute of Medicine, Szaserow 128, 04-141 Warsaw, Poland

E-mail: rdtt@wp.pl

Key words: gemcitabine, chemotherapy toxicity, polymorphism, cytidine deaminase threatening (2-4). Factors increasing GCB toxicity include its combination with platinum derivatives or taxanes, liver and kidney diseases, and alcohol abuse (5-10). There are no evidence-based and generally accepted recommendations for dose modifications of GCB, and clinical decisions are typically made based on empirical grounds.

The main enzyme involved in hepatic metabolism of GCB is cytidine deaminase (CDA), encoded by the $C D A$ gene located in locus 1p36.2-35 (11-15). Data regarding toxicity related to $C D A$ polymorphisms are inconsistent (16-18). Nevertheless, several studies demonstrated that single nucleotide polymorphism (SNP) of CDA c.79 $\mathrm{A}>\mathrm{C}$, c.208 G>A and c.435C $>\mathrm{T}$, with resulting decreased serum $\mathrm{CDA}$ concentration, may lead to severe toxicity induced by GCB (18-31).

We describe here severe toxicity in four patients treated with GCB used alone or in combination with cisplatin. For each case, the probability of adverse drug reaction probability was assessed using the Naranjo scale described in the study by Naranjo et al (32). In the search of potential toxicity causes, we performed in all cases evaluation of $C D A$ polymorphisms.

Case 1. A 67-year-old-woman was diagnosed with poorly differentiated tubule-solid gallbladder adenocarcinoma invading the liver and spreading to the greater omentum. The patient reported recurrent pain in the upper abdomen, but was otherwise in a fairly good condition [World Health Organization Performance Status (WHO PS) 2], with body mass index of 18.6 and no apparent active inflammatory symptoms. Serum alanine transaminase (ALT) and aspartate transaminase (AST) levels were 55 and $100 \mathrm{U} / 1$, respectively, and the levels of bilirubin, serum creatinine and glomerular filtration rate were within the normal values. Biochemical abnormalities included elevated levels of $\mathrm{C}$ reactive protein (CRP; $9 \mathrm{mg} / \mathrm{dl}$ ), alkaline phosphatase (ALP; $1237 \mathrm{U} / \mathrm{l}$ ), gamma-glutamyl transpeptidase (GGT; 1,073 U/1) and white blood cells (WBC; 10.4x10 $/ 1$ ). Six months earlier she underwent biliary stenting by percutaneous transhepatic cholangiography, complicated by transient paralytic ileus. Braun gastrointestinal bypass was performed one month before commencing palliative chemotherapy. She was medicated with fentanyl patch (50 g/day every 3 days) and 
acetaminophen (500 mg orally three times daily), morphine (20 mg daily) and low molecular weight heparin (60 mg once daily subcutaneously). Due to hypertension, for a few years the patient had been administered enalapril, $5 \mathrm{mg}$ twice daily. Treatment plan included intravenous administration of GCB $1,000 \mathrm{mg} / \mathrm{m}^{2}$ on day 1 and 8 , and cisplatin $25 \mathrm{mg} / \mathrm{m}^{2}$ on day 1 , every 21 days. Two days after the first administration of chemotherapy the patient developed a sudden deterioration of general condition: weakness, severe pain in the right hypochondrium and hypotension, accompanied by increased liver parameters: AST, 1876 U/1 [Common Terminology Criteria for Adverse Events v. 4 Grade (G)4]; ALT, 497 U/1 (G3); bilirubin, $2.3 \mathrm{mg} / \mathrm{dl}(\mathrm{G} 2)$; anemia $\mathrm{Hg}, 8.9 \mathrm{~g} / \mathrm{dl}(\mathrm{G} 2)$; leukopenia $\left(2.0 \times 10^{9} / 1\right)$; and neutropenia $\left(1.44 \times 10^{9} / 1\right)$. There were no ECG signs of acute myocardial ischemia, and the troponin level was $0.011 \mathrm{ng} / \mathrm{ml}$ (with a level of $\geq 0.12 \mathrm{ng} / \mathrm{ml}$ corresponding to acute myocardial infarction). Despite the treatment (hydrocortisone, isotonic solution, morphine) the patient died within $6 \mathrm{~h}$ after onset of symptoms due to acute cardio-pulmonary insufficiency. According to the will of the family, the autopsy was not performed. Based on the Naranjo scale, the drug causality of adverse reactions was considered probable (total score, 5): Are there previous conclusive reports on this reaction? Yes (1+). Did the adverse event appear after the suspected drug was administered? Yes (2+). Are there alternative causes that could on their own have caused the reaction? No (2+).

Case 2. A 60-year-old woman presented with metastatic squamous cell carcinoma of the right lung. She was a long-term cigarette smoker, with accompanying chronic obstructive pulmonary disease and carotid atherosclerosis, resulting in an episode of stroke and epilepsy. Five years earlier the patient received radical chemoradiation for laryngeal cancer and one year earlier she underwent craniotomy for brain metastasis from lung cancer, whole brain irradiation and three cycles of vinorelbine with cisplatin. At admission WHO PS was 1 and body mass index 26 . There were no overt infections, apart from asymptomatic bacteriuria. The $\mathrm{CT}$ scan showed multiple metastatic lesions in both lungs, a large metastatic mass in the right adrenal gland and enlarged abdominal lymph nodes. She was administered tramadol (50 mg twice daily), ketoprofen (100 mg daily), dexamethasone (4 mg daily) and omeprazole (20 mg daily). The complete blood count (CBC), liver and kidney function parameters were within normal values, and the only laboratory abnormalities included leukocytosis related to chronic steroid therapy.

The treatment plan included administration of GCB $1,250 \mathrm{mg} / \mathrm{m}^{2}$ on day 1 and 8 , and cisplatin $80 \mathrm{mg} / \mathrm{m}^{2}$ intravenously, every 21 days. One day after the first dose of GCB in combination with cisplatin, she developed a sudden deterioration of the general status: symptoms of pulmonary edema, liver failure and a shock. The patient was administered ceftazidime, steroids, isotonic solution, furosemide, morphine, dopamine, dobutamine, noradrenaline and synchronized intermittent mandatory ventilation. Despite this, on day 4 after the initiation of chemotherapy, she died with symptoms of acute cardio-pulmonary insufficiency. The autopsy showed lung sarcomatoid carcinoma with metastases to the right adrenal gland and paraaortic lymph nodes. There was also chronic hypertrophy of the left and right heart ventricles,

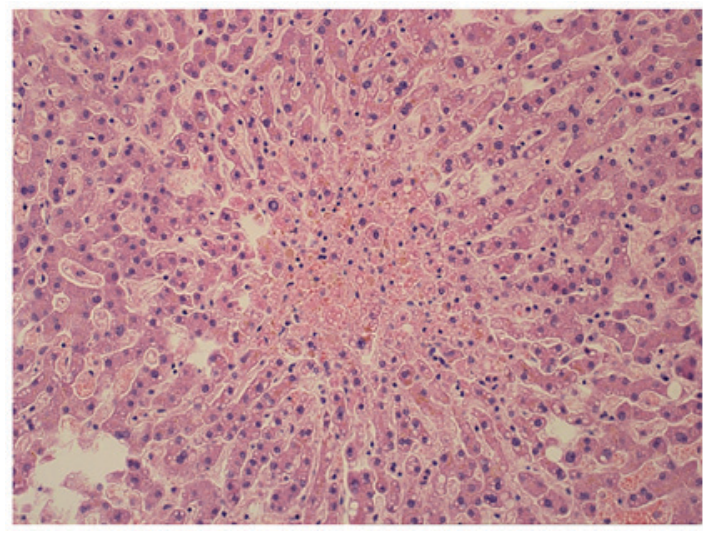

Figure 1. Hepatocyte necrosis in the centers of lobules. The foci of cell necrosis; hepatocytes with features of microvesicular degeneration and intracellular cholestasis (hematoxylin and eosin; magnification, x200).

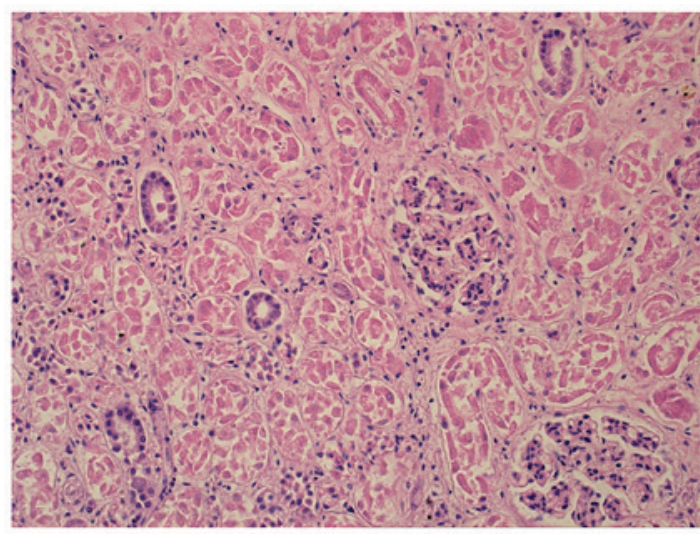

Figure 2. Acute renal tubular necrosis (eosinophilic, flattened epithelium with cell cytoplasm homogenization, exfoliative to tubular lumen; hematoxylin and eosin; magnification, x200).

liver and kidney damage, brain edema (Figs. 1 and 2), lung emphysema, atelectasis, passive congestion and bilateral bronchopneumonia. The probable cause of death was septic shock and multiple organ failure caused by severe purulent lung inflammation. Based on the Naranjo scale the drug causality of adverse reactions, were considered possible (total score, 2): Are there previous conclusive reports on this reaction? Yes (1+). Did the adverse event appear after the suspected drug was administered? Yes (2+). Are there alternative causes that could on their own have caused the reaction? Yes (-1).

Case 3. A 68 year old man was diagnosed with tumor of the pancreatic head. He presented with obstructive jaundice, with a total serum bilirubin level of $12.8 \mathrm{mg} / \mathrm{dl}$. CT scan and ultrasound showed only a stricture in common bile duct, confirmed by endoscopic retrograde cholangiopancreatography. Laparotomy revealed an unresectable tumor of pancreatic head (adenocarcinoma G2) infiltrating surrounding vessels, and Roux-Y hepaticojejunoanastomosis was performed. The patient was in good general status (WHO PS 1), with hypertension controlled with combined therapy. Due to exocrine pancreatic insufficiency, he was treated with pancreatine. Laboratory abnormalities before chemotherapy commencement included thrombocytopenia (89 G/1) and increased Ca 
CDA c.79

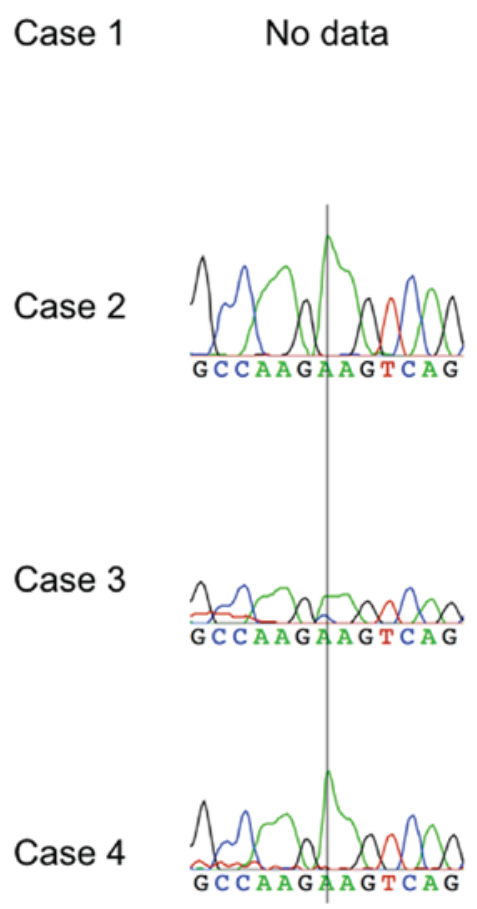

CDA c.208

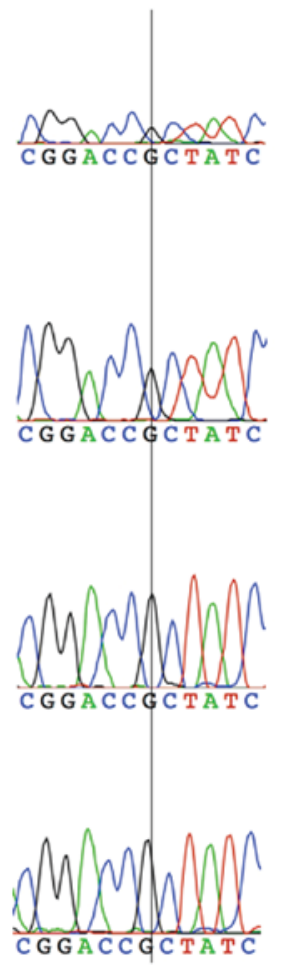

CDA c.435

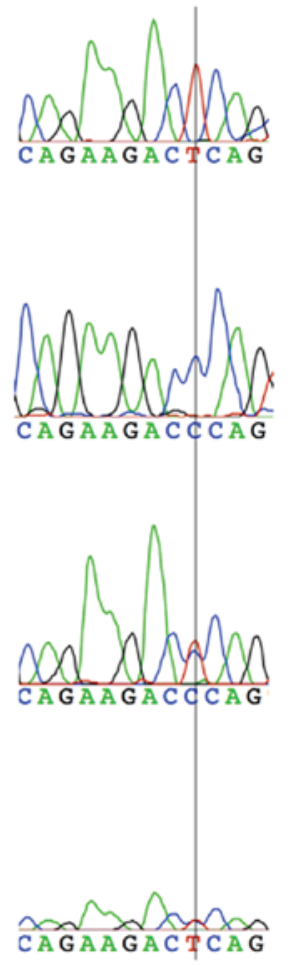

Figure 3. The evaluation of $C D A$ polymorphism: 79A $>\mathrm{C}, 208 \mathrm{G}>\mathrm{A}, 435 \mathrm{C}>\mathrm{T}$; four cases. CDA, cytidine deaminase.

19-9 level (879 U/ml). Other CBC parameters, kidney and liver functions were normal. Planned chemotherapy included GCB at a dose of $1,000 \mathrm{mg} / \mathrm{m}^{2}$ on day 1,8 and 15 , with $30 \%$ dose reduction due to baseline thrombocytopenia. One week after the first GCB administration, platelet level decreased to 32 G/1 (G3). The next drug administration, was possible only after three weeks, also with 30\% dose reduction. Again, a week later a significant thrombocytopenia (60 G/l) occurred (G2). The third GCB administration was delayed by a week, and the dose was further reduced to $50 \%$ of the due dose. Despite this, he developed a G2 thrombocytopenia $\left(67 \times 10^{9} / 1\right)$ with accompanying $\mathrm{G} 2$ neutropenia $\left(1.4 \times 10^{9} / 1\right)$ and $\mathrm{G} 2$ anemia $(8.7 \mathrm{~g} / \mathrm{dl})$. Abdominal ultrasound showed disease progression and a increasing Ca 19-9 serum level (1,707 U/ml). Owing only to the local tumor extension, the patient received radiotherapy at a dose of $50.4 \mathrm{~Gy}$ in 28 fractions without concurrent chemotherapy. Based on the Naranjo scale, the drug causality of the adverse reactions was considered probable (total score, 6): Are there previous conclusive reports on this reaction? Yes (1+). Did the adverse event appear after the suspected drug was administered? Yes $(2+)$. Did the adverse reaction improve when the drug was discontinued? Yes (1+). Did the adverse event reappear when the drug was re-administered? Yes (2+). Are there alternative causes that could on their own have caused the reaction? Yes (-1). Was the reaction more severe when the dose was increased or less severe when the dose was decreased? Yes (1+).

Case 4. A 58 year old woman was diagnosed with a tumor of the pancreatic head. The first symptom was mechanical jaundice, with serum bilirubin level of $13,5 \mathrm{mg} / \mathrm{dl}$. Abdominal $\mathrm{CT}$ revealed a $2 \mathrm{~cm}$ mass of the pancreatic head. Endoscopic retrograde cholangiopancreatography showed a stenosis of the common bile and pancreatic ducts, which was managed by stent insertion. A month later a radical pancreatoduedenectomy was performed. Pathology examination showed G1 ductal adenocarcinoma staged pT3N0M0. There was blood and lymphatic vessels invasion and no tumor-free margins were obtained. After the surgery the patient was in a general good condition (WHO PS 1), with hypertension treated with amlodypine. CBC parameters, kidney and liver functions were within normal ranges. The patient received single-agent adjuvant GCB at a dose of $1,000 \mathrm{mg} / \mathrm{m}^{2}$ on days 1,8 and 15. Two days after the third administration of GCB she developed a sudden deterioration of general status: weakness, severe pain in the right hypochondrium, vomiting (G2) and fever (G2). Blood tests showed increased bilirubin level $(1.9 \mathrm{mg} / \mathrm{dl})$, leukocytosis $\left(14 \times 10^{9} / \mathrm{l}\right)$, neutrocytosis $\left(11 \times 10^{9} / \mathrm{l}\right)$, anemia $(\mathrm{Hg}, 8.1 \mathrm{~g} / \mathrm{dl})$, elevated CRP (228 mg/l) and procalcitonin $(1.0 \mathrm{ng} / \mathrm{ml})$. Microbiological tests were negative. The patient was administered antifungals, antipyretics, setrons, intravenous fluids and electrolyte supplementation, the general condition improved, and after three weeks the patient began a second chemotherapy cycle. Shortly after the first GCB administration she developed a severe pain in the upper abdomen, accompanied by a headache and fever (G2). The patient developed G3 neutropenia $\left(0.68 \times 10^{9} / 1\right)$ and was treated with oral antibiotics. Due to repeating toxicity, chemotherapy was discontinued. Abdominal CT showed a few small liver metastases. She received five cycles of chemotherapy including oxaliplatin, 5-fluorouracil and leucovorine (OFF), which was well tolerated and resulted in a complete remission. After 2.5 years, due to local progression, OFF chemotherapy was reinstituted and now, 3.5 years after the initial treatment, the 
patient developed a metastatic lesion in sigmoid colon, and was administered palliative radiotherapy. Despite this, she remains in good general condition (PS 1). Based on the Naranjo scale, the drug causality of adverse reactions was considered probable (total score, 5) Are there previous conclusive reports on this reaction? Yes (1+). Did the adverse event appear after the suspected drug was administered? Yes $(2+)$. Did the adverse reaction improve when the drug was discontinued? Yes $(+1)$. Did the adverse event reappear when the drug was re-administered? Yes (2+). Are there alternative causes that could on their own have caused the reaction? Yes (-1).

Polymorphism of CDA gene assessment. In a search for the cause of the severe toxicity, in all cases we assessed three SNPs of $C D A$ gene related to GCB metabolism: c.79A $>C$ (rs2072671), c. 208G $>$ A (rs60369023) and c.435C $>$ T (rs1048977). In the two deceased cases DNA was obtained from paraffin embedded healthy tissue material. Paraffin was removed with xylene and isolation was done using the Cobas DNA Sample Preparation Kit, according to the procedure specified by the manufacturer (Roche Diagnostic, Warsaw, Poland). In another two patients DNA was isolated from peripheral blood samples using Genomic Midi Ax (A\&A Biotechnology, Gdynia, Poland). All CDA polymorphisms were analyzed by polymerase chain reaction (PCR) followed by bidirectional Sanger sequencing. Sequences were analyzed using the reference sequence CDA (NM_001785.2) and Sequencher software version 4.10.1 (Gene Codes Corporation, Ann Arbor, MI, USA). The genotyping results are presented in Fig. 3. In the case 1, the presence of a homozygous $C D A$ variant c.435TT was found while the reaction product PCR was not obtained for amplicon including polymorphism c.79A $>$ C. Cases 3 and 4 showed a heterozygous variant CDA c.435CT, accompanied in case 3 by a heterozygotic c.79A >C variant. In no patient the c. $208 \mathrm{G}>\mathrm{A}$ variant was diagnosed.

\section{Discussion}

We report here four severe toxicity cases, including two fatal, following the administration of GCB alone or in combination with cisplatin. In three of these cases the causative role of GCB therapy was considered probable and in another one as possible. In the differential diagnosis all risk factors present in patients at start of therapy were included. Patient 1, 6 months earlier underwent biliary stenting by percutaneous transhepatic cholangiography, complicated by transient paralytic ileus, with persistently elevated but stable ALP and gamma-glutamyl transpeptidase. In patients 2 and 4 an infection, although not diagnosed, could be the main cause of sudden deterioration, but with strong association with GCB administration. In patient 4 , good tolerance of other cytotoxic agents suggest causative role of GCB. Patient 3 presented with initial trombocytopenia, which contributed to subsequent toxicity.

The two fatal cases in this series have some common features: concomitant administration of GCB and cisplatin, and sudden onset after the start of chemotherapy (two days; case 1, and one day; case 2). The combination of GCB and cisplatin is routinely used in various malignancies including NSCLC and advanced biliary cancer, and is considered safe. However there are data indicating that this regimen may occasionally induce oxidative stress leading to multi-organ failure $(2,5,33,34)$. Cisplatin-induced liver toxicity has been attributed to the enhanced expression of cytochrome P450 2E1 (CYP2E1), and is exacerbated in patients with diabetes, obesity, nicotine addiction or alcohol abuse (34,35). Several preclinical studies have investigated possibilities of hepatic mitochondrial oxidative damage protection using selenium, vitamin E, a hydroxyl radical scavenger dimethylthiourea (DMTU) and a polyphenolic flavonoid daidzein (36-38), but none has yet found its application in clinical practice.

Data on the relationship between $C D A$ polymorphisms and GCB toxicity are inconsistent (15-31). Severe GCB toxicity in NSCLC patients was reported in cases with heterozygous c.437CT variant and, to a lesser degree, in those with homozygous c.435TT variant $(25,26)$. Interestingly, the latter was also found to be related to better response to GCB treatment, owing to reduced serum CDA concentration and higher exposure to active GCB metabolites $(27,28)$. Heterozygous c.79A $>C$ polymorphism was reported to cause severe leukopenia and neutropenia $(14,19-22)$.

In our series, c.435TT variant was found in one patient, and c.435CT variant in two (in one accompanied by c.79A $>\mathrm{C}$ variant). In one case the presence of c.79A $>C$ could not be excluded, due to inability of performing the PCR reaction. No case showed the presence of c. $208 \mathrm{G}>\mathrm{A}$ variant, which is typical for the Asian population $(15,23,24)$. Importantly, our analysis included only three most common $C D A$ polymorphisms, therefore the presence of other, less frequent variants with potential impact on GCB metabolism cannot be excluded $(15,21,39)$. Previous studies suggested that CDA deficiency associated with $C D A$ gene polymorphisms may affect more patients treated with GCB, but the lack of population data does not allow for the estimation of real $C D A$ polymorphisms frequency. In patients with decreased CDA activity in the serum, the risk of serious side effects is in the range of $5-10 \%$ for GCB alone and $15-30 \%$ for GCB combinations $(27,28)$. The evaluation of gene polymorphisms is relatively simple and non-invasive (swabbing the inner site of the cheek), yet currently there are no recommendations for routine pretreatment assessment of CDA polymorphisms or CDA serum level $(18,40,41)$.

In conclusion, determining the cause of acute $\mathrm{GCB}$ toxicity, including both baseline clinical conditions and genetic susceptibilities, may inform clinical decisions. In patients with clinical factors predisposing to increased risk of toxicity, assessment of polymorphic variants related to pyrimidine metabolism may increase treatment safety.

\section{Acknowledgements}

The authors thank Ms. Claudia Wiewiora for linguistic check.

\section{References}

1. Toschi L, Finocchiaro G, Bartolini S, Gioia V and Cappuzzo F: Role of gemcitabine in cancer therapy. Future Oncol 1: 7-17, 2005.

2. Aapro MS, Martin C and Hatty S: Gemcitabine-a safety review. Anticancer Drugs 9: 191-201, 1998.

3. Tonato M, Mosconi AM and Martin C: Safety profile of gemcitabine. Anticancer Drugs 6 (Suppl 6): S27-S32, 1995.

4. Wong A, Soo RA, Yong WP and Innocenti F: Clinical pharmacology and pharmacogenetics of gemcitabine. Drug Metab Rev 41: 77-88, 2009. 
5. van Moorsel CJ, Pinedo HM, Veerman G, Bergman AM, Kuiper CM, Vermorken JB, van der Vijgh WJ and Peters GJ: Mechanisms of synergism between cisplatin and gemcitabine in ovarian and non-small-cell lung cancer cell lines. Br J Cancer 80 981-990, 1999.

6. Shord SS, Faucette SR, Gillenwater HH, Pescatore SL, Hawke RL, Socinski MA and Lindley C: Gemcitabine pharmacokinetics and interaction with paclitaxel in patients with advanced non-small-cell lung cancer. Cancer Chemother Pharmacol 51: 328-336, 2003

7. Jiang X, Galettis P, Links M, Mitchell PL and McLachlan AJ: Population pharmacokinetics of gemcitabine and its metabolite in patients with cancer: Effect of oxaliplatin and infusion rate. $\mathrm{Br}$ J Clin Pharmacol 65: 326-333, 2008.

8. Teusink AC and Hall PD: Toxicities of gemcitabine in patients with severe hepatic dysfunction. Ann Pharmacother 44: 750-754, 2010.

9. Felici A, Di Segni S, Milella M, Colantonio S, Sperduti I, Nuvoli B, Contestabile M, Sacconi A, Zaratti M, Citro G and Cognetti F: Pharmacokinetics of gemcitabine at fixed-dose rate infusion in patients with normal and impaired hepatic function. Clin Pharmacokinet 48: 131-141, 2009.

10. Ciccolini J, Serdjebi C, Peters GJ and Giovannetti E: Pharmacokinetics and pharmacogenetics of gemcitabine as a mainstay in adult and pediatric oncology: An EORTC-PAMM perspective. Cancer Chemother Pharmacol 78: 1-12, 2016.

11. Demontis S, Terao M, Brivio M, Zanotta S, Bruschi M and Garattini E: Isolation and characterization of the gene coding for human cytidine deaminase. Biochim Biophys Acta 1443: 323-333, 1998

12. Gilbert JA, Salavaggione OE, Ji Y, Pelleymounter LL, Eckloff BW, Wieben ED, Ames MM and Weinshilboum RM: Gemcitabine pharmacogenomics: Cytidine deaminase and deoxycytidylate deaminase gene resequencing and functional genomics. Clin Cancer Res 12: 1794-1803, 2006.

13. Kocabas NA, Aksoy P, Pelleymounter LL, Moon I, Ryu JS, Gilbert JA, Salavaggione OE, Eckloff BW, Wieben ED, Yee V, et al: Gemcitabine pharmacogenomics: Deoxycytidine kinase and cytidylate kinase gene resequencing and functional genomics. Drug Metab Dispos 36: 1951-1959, 2008.

14. Xu J, Zhou Y, Zhang J, Chen Y, Zhuang R, Liu T and Cai W: High incidence of severe neutropenia after gemcitabine-based chemotherapy in Chinese cancer patients with CDA 79A $>C$ mutation. Clin Chim Acta 413: 1284-1287, 2012.

15. Micozzi D, Carpi FM, Pucciarelli S, Polzonetti V, Polidori P, Vilar S, Williams B, Costanzi S and Vincenzetti S: Human cytidine deaminase: A biochemical characterization of its naturally occurring variants. Int J Biol Macromol 63: 64-74, 2014.

16. Serdjebi C, Milano G and Ciccolini J: Role of cytidine deaminase in toxicity and efficacy of nucleosidic analogs. Expert Opin Drug Metab Toxicol 11: 665-672, 2015.

17. Yoon KA, Woo SM, Hong EK, Jung MK, Park WS, Bae K, Han SS, Kim TH, Koh YH, Park SJ and Lee WJ: Cytidine deaminase as a molecular predictor of gemcitabine response in patients with biliary tract cancer. Oncology 89: 345-350, 2015.

18. Evrard A and Mbatchi L: Genetic polymorphisms of drug metabolizing enzymes and transporters: The long way from bench to bedside. Curr Top Med Chem 12: 1720-1729, 2012.

19. Joerger M, Burgers SA, Baas P, Smit EF, Haitjema TJ, Bard MP, Doodeman VD, Smits PH, Vincent A and Huitema AD: Germline polymorphisms in patients with advanced nonsmall cell lung cancer receiving first-line platinum-gemcitabine chemotherapy: A prospective clinical study. Cancer 118: 2466-2475, 2012.

20. Tanaka M, Javle M, Dong X, Eng C, Abbruzzese JL and Li D: Gemcitabine metabolic and transporter gene polymorphisms are associated with drug toxicity and efficacy in patients with locally advanced pancreatic cancer. Cancer 116: 5325-5335, 2010.

21. Tibaldi C, Giovannetti E, Vasile E, Mey V, Laan AC, Nannizzi S, Di Marsico R, Antonuzzo A, Orlandini C, Ricciardi S, et al: Correlation of CDA, ERCC1, and XPD polymorphisms with response and survival in gemcitabine/cisplatin-treated advanced non-small cell lung cancer patients. Clin Cancer Res 14: 1797-1803, 2008.

22. Farrell JJ,Bae K, Wong J, Guha C,Dicker APand Elsaleh H: Cytidine deaminase single-nucleotide polymorphism is predictive of toxicity from gemcitabine in patients with pancreatic cancer: RTOG 9704. Pharmacogenomics J 12: 395-403, 2012.

23. Yomemori K, Ueno H, Okusaka T, Yamamoto N, Ikeda M, Saijo N, Yoshida T, Ishii H, Furuse J, Sugiyama E, et al: Severe drug toxicity associated with a single-nucleotide polymorphism of the cytidine deaminase gene in a Japanes cancer patient treated with gemcytabine plus cisplatin. Clin Cancer Res 11: 2620-2624, 2005.
24. Sugiyama E, Kaniwa N, Kim SR, Kikura-Hanajiri R, Hasegawa R, Maekawa K, Saito Y, Ozawa S, Sawada J, Kamatani N, et al: Pharmacokinetics of gemcitabine in Japanese cancer patients: The impact of a cytidine deaminase polymorphism. J Clin Oncol 25: 32-42, 2007.

25. Ludovini V, Floriani I, Pistola L, Minotti V, Meacci M, Chiari R, Garavaglia D, Tofanetti FR, Flacco A, Siggillino A, et al: Association of cytidine deaminase and xeroderma pigmentosum group D polymorphisms with response, toxicity, and survival in cisplatin/gemcitabine-treated advanced non-small cell lung cancer patients. J Thorac Oncol 6: 2018-2026, 2011.

26. Okazaki T, Javle M, Tanaka M, Abbruzzese JL and Li D: Single nucleotide polymorphisms of gemcitabine metabolic genes and pancreatic cancer survival and drug toxicity. Clin Cancer Res 16: 320-329, 2010.

27. Ciccolini J, Dahan L, André N, Evrard A, Duluc M, Blesius A, Yang C, Giacometti S, Brunet C, Raynal C, et al: Cytidine deaminase residual activity in serum is a predictive marker of early severe toxicities in adults after gemcitabine-based chemotherapies. J Clin Oncol 28: 160-165, 2010.

28. Mercier C, Raynal C, Dahan L, Ortiz A, Evrard A, Dupuis C, Blesius A, Duluc M, Franceschini F, Giacometti S, et al: Toxic death case in a patient undergoing gemcitabine-based chemotherapy in relation with cytidine deaminase downregulation. Pharmacogenet Genomics 17: 841-844, 2007.

29. Ding X, Chen W, Fan H and Zhu B: Cytidine deaminase polymorphism predicts toxicity of gemcitabine-based chemotherapy. Gene 559: 31-37, 2015.

30. Zhou M, Ding YJ, Feng Y, Zhang QR, Xiang Y and Wan HY: Association of xeroderma pigmentosum group D (Asp312Asn, Lys751Gln) and cytidine deaminase (Lys27Gln, Ala70Thr) polymorphisms with outcome in Chinese non-small cell lung cancer patients treated with cisplatin-gemcitabine. Genet Mol Res 13: 3310-3318, 2014

31. Li H, Wang $\mathrm{X}$ and Wang $\mathrm{X}$ : The impact of CDA A79C gene polymorphisms on the response and hematologic toxicity in gemcitabine-treated patients: A meta-analysis. Int J Biol Markers 29: e224-e232, 2014

32. Naranjo CA, Busto U, Sellers EM, Sandor P, Ruiz I, Roberts EA, Janecek E, Domecq C and Greenblatt DJ: A method for estimating the probability of adverse drug reactions. Clin Pharmacol Ther 30: 239-245, 1981 .

33. Waseem M, Bhardwaj M, Tabassum H, Raisuddin S and Parvez S: Cisplatin hepatotoxicity mediated by mitochondrial stress. Drug Chem Toxicol 38: 452-459, 2015.

34. Dasari S and Tchounwou PB: Cisplatin in cancer therapy: Molecular mechanisms of action. Eur J Pharmacol 740: 364-378, 2014.

35. Lu Y and Cederbaum AI: Cisplatin-induced hepatotoxicity is enhanced by elevated expression of cytochrome P450 2E1. Toxicol Sci 89: 515-523, 2006.

36. Karale S and Kamath JV: Effect of daidzein on cisplatin-induced hematotoxicity and hepatotoxicity in experimental rats. Indian J Pharmacol 49: 49-54, 2017

37. Naziroglu M, Karaoğlu A and Aksoy AO: Selenium and high dose vitamin $\mathrm{E}$ administration protects cisplatin-induced oxidative damage to renal, liver and lens tissues in rats. Toxicology 195: 221-230, 2004

38. dos Santos NA, Martins NM, Curti C, Pires Bianchi Mde L and dos Santos AC: Dimethylthiourea protects against mitochondrial oxidative damage induced by cisplatin in liver of rats. Chem Biol Interact 170: 177-186, 2007.

39. Raynal C, Ciccolini J, Mercier C, Boyer JC, Polge A, Lallemant B, Mouzat K, Lumbroso S, Brouillet JP and Evrard A: High-resolution melting analysis of sequence variations in the cytidine deaminase gene (CDA) in patients with cancer treated with gemcitabine. Ther Drug Monit 32: 53-60, 2010.

40. Petros WP and Evans WE: Pharmacogenomics in cancer therapy: Is host genome variability important? Trends Pharmacol Sci 25: 457-464, 2004

41. Evrard A, Lacarelle B and Ciccolini J: Severe or lethal toxicities with nucleosidic analogs: Time for action. Pharmacogenomics 14: 227-230, 2013. 